\title{
Magnetism of pristine Fe films on $\operatorname{GaAs}(100)$
}

\author{
Jae-Min Lee, ${ }^{1}$ J.-Y. Kim, ${ }^{2}$ S.-U. Yang, ${ }^{3}$ B.-G. Park, ${ }^{4}$ J.-H. Park, ${ }^{4}$ S.-J. Oh, ${ }^{1}$ and J.-S. Kim ${ }^{3}, *$ \\ ${ }^{1}$ Department of Physics and Astronomy and Center for Strongly Correlated Material Research, Seoul National University, \\ Seoul 151-747, Korea \\ ${ }^{2}$ Pohang Accelerator Laboratory, Pohang University of Science and Technology, Pohang 790-784, Korea \\ ${ }^{3}$ Department of Physics, Sook-Myung Women's University, Seoul 140-742, Korea \\ ${ }^{4}$ Department of Physics, Pohang University of Science and Technology, Pohang 790-784, Korea \\ (Received 15 May 2007; revised manuscript received 18 June 2007; published 16 August 2007)
}

\begin{abstract}
We grew Fe films on $\mathrm{GaAs}(100)$ at $\sim 80 \mathrm{~K}$ to suppress interface alloying and As outdiffusion, and obtained kinetically stabilized, pristine Fe films. We studied their magnetic properties using both X-ray magnetic circular dichroism and the magneto-optic Kerr effect. The salient magnetic features are as follows. A 2.1-ML-thick Fe film is already ferromagnetic and exhibits perpendicular magnetic anisotropy (PMA). Moreover, a spin reorientation transition occurs in films with $\sim 2.8$ monolayer thickness. The PMA is attributable to preservation of the surface magnetic anisotropy by suppressing the As outdiffusion during the low-temperature growth of the Fe film. The PMA is associated with the enhancement of the orbital moment by only $\sim 50 \%$ in contrast to values reported in some previous studies.
\end{abstract}

DOI: 10.1103/PhysRevB.76.052406

PACS number(s): 75.70.Cn, 72.25.Mk, 79.60.Jv

\section{INTRODUCTION}

Heteroepitaxial systems composed of ferromagnetic metal and semiconductors have attracted considerable attention because they can be used as the platform for developing magnetoelectronic devices that exploit both the magnetic and electronic degrees of freedom. Fe films on GaAs substrates have been a focus of such attention due to their desirable physical properties for magnetoelectronic applications, e.g, both the high spin moment and high Curie temperature of Fe, and the high electronic mobility of GaAs. Furthermore, Fe films can be grown epitaxially on GaAs substrates because of their crystallographic similarity. ${ }^{1}$ This was the first system in which spin injection from a ferromagnetic metal to a semiconductor was achieved at room temperature. ${ }^{2}$ It has thus drawn renewed attention as a prototype for the study of spin injection and related modern magnetoelectronic applications, a field often called spintronics.

Despite their great promise, Fe films on GaAs substrates have a serious drawback. The outdiffusion of $\mathrm{Ga}$ and As from the substrate to the Fe film occurs on a large scale, and this results in the formation of Fe compounds in the film and in a segregated As layer on the Fe film. ${ }^{3,4}$ The formation of such compounds and the As segregation are expected to reduce the magnetic moment of the Fe film significantly, and possibly even quench it depending on the number of As neighbors for each Fe atom., Experimental results are inconsistent with respect to the existence of the magnetically dead layer, the ferromagnetic onset coverage, ${ }^{7-11}$ and the direction of the in-plane magnetic easy axis. ${ }^{12-14}$ To a certain extent, the discrepancies can be attributed to different sample preparation conditions, different surface orientations, and surface terminations that give rise to different degrees of outdiffusion of Ga and As. Hence, the growth and characterization of pristine Fe films on the GaAs substrate still remain a major impediment to determining the true magnetic properties of the Fe film.

Various efforts have been made to suppress the outdiffusion of both $\mathrm{Ga}$ and As, including the passivation of the
GaAs surface by interlayers of $\mathrm{S},{ }^{15} \mathrm{Er},{ }^{16}$ and $\mathrm{Al} .{ }^{17}$ However, all these previous attempts have achieved only limited success, especially in blocking the As segregation, which remains a serious challenge. Moreover, the passivating layers themselves are foreign to the Fe film and would seriously modify its magnetic and transport properties, especially near the interface. The inability to grow the pristine Fe film on GaAs has even encouraged the search for the other ferromagnet/semiconductor systems such as Fe/ZnSe(100) (Ref. 18) and Fe/InAs(100). ${ }^{19}$

Recently, some of the present authors have found a solution to the longstanding problem of growing pure Fe films on GaAs(100) or kinetic stabilization through low growth temperatures of less than $130 \mathrm{~K}$. Using both photoelectron spectroscopy $^{20}$ and X-ray reflectivity (XRR), ${ }^{21}$ we have confirmed that almost complete suppression of both the compound formation and the As surface segregation is possible. In this paper, we report the magnetic properties of such a kinetically stabilized, virtually pristine Fe film on GaAs(100) that has been studied by both X-ray magnetic circular dichroism (XMCD) and the magneto-optic Kerr effect (MOKE). We have observed two salient magnetic features of the $\mathrm{Fe}$ films. First, an $\sim 2.1$ monolayer (ML) Fe film on GaAs(100) is ferromagnetic and exhibits perpendicular magnetic anisotropy (PMA). Second, in an $\sim 2.8 \mathrm{ML}$ Fe film, a spin reorientation transition (SRT) from PMA to in-plane anisotropy occurs. Note that in sharp contrast to some previous reports, ${ }^{10,11}$ the observed PMA is not associated with any anomalously enhanced orbital moment.

\section{EXPERIMENT}

The growth and magnetic characterization of the Fe films were performed in situ in two separate ultrahigh vacuum chambers, one for the XMCD study and the other for the MOKE study. During the growth of the Fe film and magnetic measurements, the pressures of the two chambers were maintained at $\sim 2 \times 10^{-10}$ Torr for the XMCD measurement and 
(a)

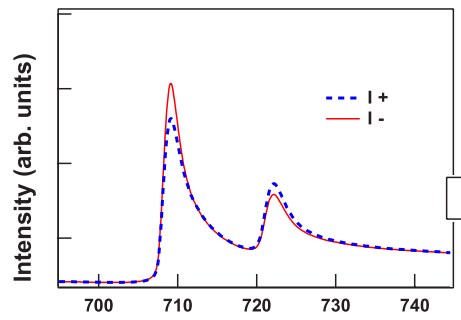

( b )

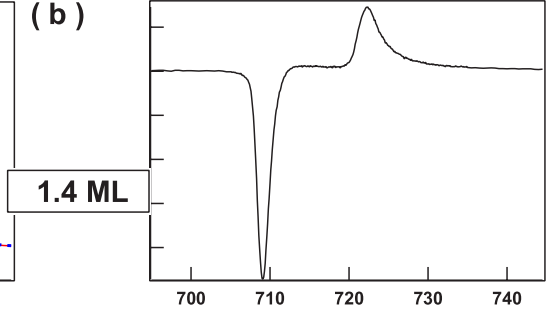

(c)

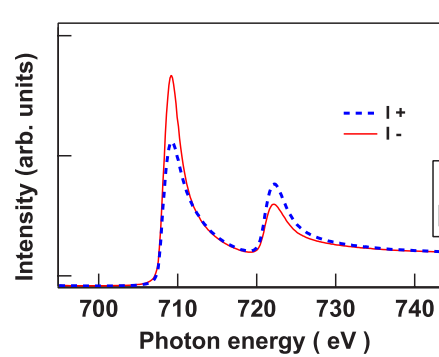

(d)

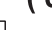

$(d)$

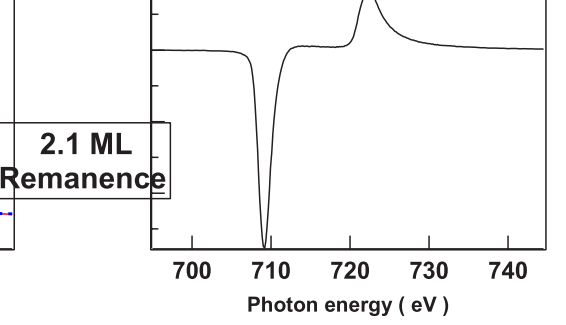

FIG. 1. (Color online) [(a) and (b)] XAS and $\mathrm{XMCD}$ of the 1.4-ML-thick Fe film under an external magnetic field of $0.5 \mathrm{~T}$. [(c) and (d)] XAS and XMCD of the 2.1-ML-thick Fe film at remanence. All the spectra are taken in the polar geometry. $I+$ and $I-$ signify two opposite magnetic field directions. $\sim 5 \times 10^{-10}$ Torr for the MOKE measurement. As a substrate, we used $\mathrm{GaAs}(100)$ samples, which were Si-doped $n$-type semiconductors. Repeated cycles of sputtering and annealing of the sample produced a clean and well ordered GaAs substrate. Annealing was performed at $570{ }^{\circ} \mathrm{C}$ for $30 \mathrm{~min}$. The clean surface showed a well-defined $4 \times 2$ low energy electron diffraction pattern.

The Fe film was deposited using a commercial e-beam evaporator. The deposition rate was $0.67 \AA / \mathrm{min}$. The deposition rate was estimated by deposition flux, which was carefully calibrated by both a quartz microbalance and surface $\mathrm{x}$-ray reflectivity measurements. The temperature of the substrate was maintained at $\sim 80 \mathrm{~K}$ with liquid nitrogen throughout the $\mathrm{Fe}$ deposition and the measurements of XMCD and MOKE. The temperature of the sample was determined by both a silicon diode during cooling and an IR pyrometer during annealing. According to our in situ XRR study, ${ }^{21} \mathrm{Fe}$ films on the cold substrate do not grow in a layerby-layer fashion. Thus, the coverages referred to are nominal values.

The XMCD experiments were performed at the EPU6 (2A) elliptically polarized undulator beamline at the Pohang Synchrotron Light Source in Korea. The polarization of the circularly polarized photon was about $95 \%$. The x-ray absorption spectroscopy (XAS) spectrum was obtained in the total electron yield mode by monitoring the sample drain current. An electromagnet with its maximum field $\left(H_{m}\right)$ $\sim 0.5 \mathrm{~T}$ was utilized for the XMCD measurements. The MOKE measurement was performed by employing an intensity-stabilized He-Ne laser and four-pole magnet with $H_{m} \sim 0.1 \mathrm{~T}$. For both the experiments, the in-plane direction was chosen to be [011], which is generally accepted as the in-plane easy axis of the Fe film. ${ }^{12,14}$

\section{RESULTS}

For a 1.4-ML-thick Fe film, a very well-defined, strong dichroic signal was observed under an external magnetic field in the polar geometry, as shown in Figs. 1(a) and 1(b). For this film, however, the dichroic signal at remanence was very small compared to the saturation moment, telling that the ferromagnetic Curie temperature $\left(T_{C}\right)$ for most parts of the film was still lower than $80 \mathrm{~K}$. For a 2.1-ML-thick film, however, a well-defined dichroic signal was observed even at remanence in the polar geometry, indicating the onset of ferromagnetism [Figs. 1(c) and 1(d)].

For the study of the magnetic anisotropy, we obtained the MOKE hysteresis curves for the Fe films. In Fig. 2(a), for the 2.1-ML-thick Fe film, the polar hysteresis shows a relatively low coercive field $\left(H_{c}\right)(\sim 45 \mathrm{Oe})$ and clear remanent magnetization, while the longitudinal hysteresis shows distinc-

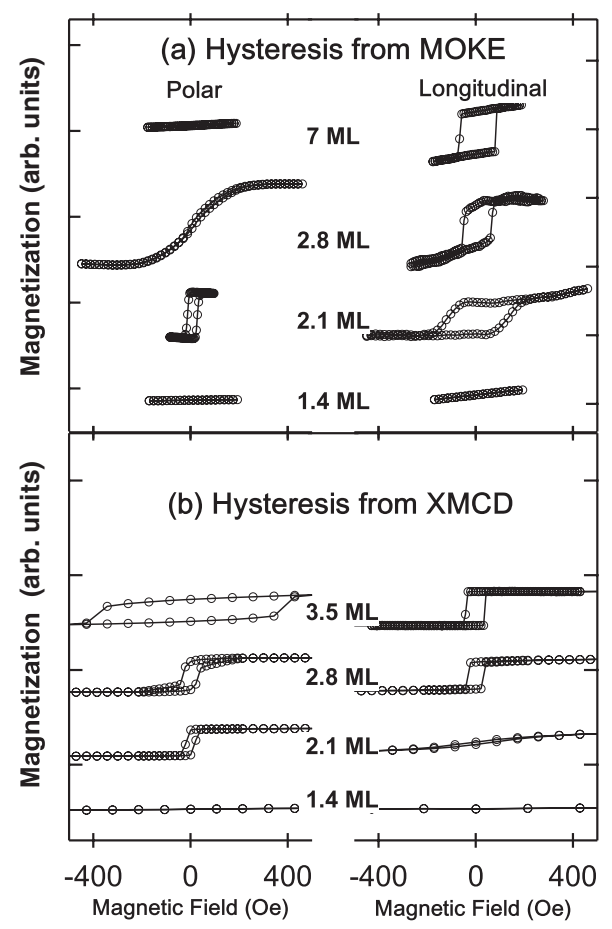

FIG. 2. (a) MOKE and (b) XMCD hysteresis curves for the Fe films with increasing Fe thickness. In the polar (longitudinal) geometry, the external magnetic field is along the [100] ([110]) direction. 


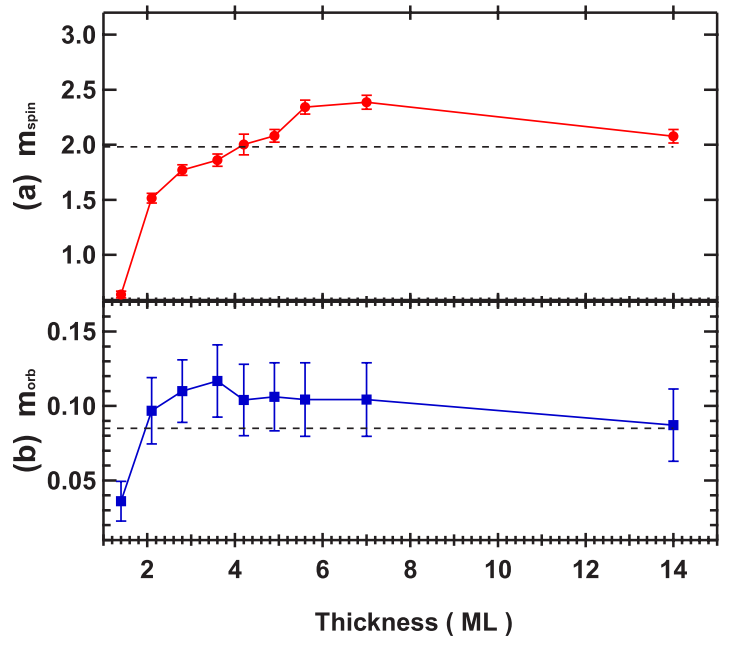

FIG. 3. (Color online) Spin and orbital moments as a function of the Fe thickness. Each moment is obtained from the XMCD spectra taken under an external magnetic field in the polar geometry. In each figure, the horizontal dashed line signifies the respective bulk value.

tively higher $H_{c}$ with poor squareness. This indicates that the 2.1-ML-thick Fe film was ferromagnetic and exhibited PMA. For the 2.8-ML-thick Fe film, the magnetic easy axis was then oriented in the in-plane direction; the SRT was complete at 2.8 ML. The hystereses obtained from the XMCD spectra with a varying magnetic field showed a similar SRT, confirming the observations from the MOKE measurement [Fig. 2(b)]. Here, we also note PMA for 2.1-ML-thick film and SRT occurring around 2.8-ML-thick film. Slightly delayed SRT in XMCD hystereses may be caused by fluctuation of deposition flux.

To obtain quantitative information on both the spin and orbital magnetic moments of Fe film, we applied the sum rule $^{23-25}$ to the XMCD and x-ray absorption spectra of $\mathrm{Fe}$ $L_{2,3}$ taken under an external magnetic field of $0.5 \mathrm{~T}$ in the polar geometry.

The sum rule reads as follows:

$$
\begin{gathered}
m_{\text {orb }}=-\frac{4 q}{3 r}\left(10-n_{3 d}\right) \frac{1}{\cos \theta} \frac{1}{0.95}, \\
m_{\text {spin }}=-\frac{6 p-4 q}{r}\left(10-n_{3 d}\right) \frac{1}{\cos \theta} \frac{1}{0.95},
\end{gathered}
$$

where $p$ and $q$ are the integral for the $L_{3}$ edge and the sum of the integral for the $L_{3}$, and $L_{2}$ edges, respectively, in the $\mathrm{XMCD} ; r$ is the sum of the integral for $L_{3}$ and $L_{2}$ in XAS; $n_{3 d}$ is the $3 d$ electron occupation number $\left[n_{3 d=\mathrm{Fe}}=6.69\right.$ (Ref. 25)]; $\theta$ is the angle between the magnet and the photon beam ( $30^{\circ}$ in our experimental setup); and 0.95 is the degree of polarization of an incident photon. Some ambiguity occurs in applying the sum rule, for example, in choosing the intervals for the integration of relevant signals for $p, q$, and $r$ values, and in subtracting background. The error bar for each data point in Fig. 3 is set by the maximum variation of orbital and spin moments from these ambiguities in the data analysis.
In Fig. 3(a), the spin moment per Fe atom $m_{s}$ gradually increases with the thickness and reaches the bulk spin moment of $\mathrm{Fe}, 1.98 \mu_{B}\left(\mu_{s}^{b u l k}\right),{ }^{25}$ for 3.5-ML-thick film. Claydon et al. ${ }^{11}$ however, reported a bulklike spin moment even for a submonolayer Fe film with thick Co-capping layer. For the Fe films in this study with a thickness smaller than $\sim 3.5 \mathrm{ML}$, the sample temperature may be higher than their $T_{C}$ 's, and/or the applied magnetic field may not be strong enough to saturate the magnetic moment.

For Fe films with a thickness in the range 3.5-7 ML, $m_{s}$ is slightly larger than $\mu_{s}^{\text {bulk }}$. One possible reason for the enhanced $m_{s}$ is that due to the low-temperature growth, the $\mathrm{Fe}$ film is composed of small islands that have a large density of low-coordinated atoms such as those forming edges. These Fe atoms would have an enhanced spin magnetic moment. Claydon et al. ${ }^{11}$ also observed enhanced spin magnetic moment for Fe films on GaAs(100) whose thickness, 4 ML, was below the coalescence regime of the islands. When the thickness of the film reached the upper limit of $14 \mathrm{ML}, m_{s}$ became almost identical to $\mu_{s}^{b u l k}$, as would be expected for bulklike film.

In Fig. 3(b), the orbital magnetic moment $m_{o}$ shows a similar thickness dependence; it exhibits an initial monotonic increase followed by a subsequent slow decrease, eventually to the point of the bulk moment. The initial monotonic increase in $m_{o}$ is attributable mainly to the undersaturation due to low $T_{C}$ and/or a low applied magnetic field. Still, films with thicknesses ranging from 2.1 to $7 \mathrm{ML}$ show larger $m_{o}$ than that of bulk, $0.085 \mu_{B}\left(\mu_{o}^{b u l k}\right)$. The enhancement factor $\left(m_{o}-\mu_{o}^{\text {bulk }}\right) / \mu_{o}^{\text {bulk }}$ is, however, $\sim 50 \%$ for the present system, which is much lower than the figure of $\sim 200 \%$ reported by Claydon et al. ${ }^{11}$

\section{DISCUSSION}

The most salient feature of the present Fe films is the presence of PMA. Previously, only in-plane magnetic anisotropy had been observed for Fe films on $\operatorname{GaAs}(100)$. $^{10,12,13,22}$ Furthermore, a recent theoretical work even predicted the absence of PMA in Fe films on GaAs(100). ${ }^{26}$ The most notable difference between the present experiment and previous ones is the low-temperature growth of the Fe film. This indicates that the main origin of the observed PMA is the preservation of the As-free surface during the growth of the film, which produces sufficient surface magnetic anisotropy to support PMA. Moreover, the chemical and structural disorder in the interface possibly due to residual interfacial reaction and low-temperature growth may lower in-plane magnetic anisotropy ${ }^{27}$ and help to observe PMA for the present system.

For the 2.1-ML-thick Fe film in which PMA was observed, the orbital moment of Fe showed only an $\sim 50 \%$ enhancement from the corresponding bulk value, which is in sharp contrast to the $200 \%$ enhancement observed for Fe films grown on $\mathrm{GaAs}(100)$ near room temperature. ${ }^{11}$ Despite the large anomalous orbital moment, those films never showed PMA regardless of the film thickness, ${ }^{28}$ and furthermore the enhanced moment was about the same regardless of the thickness of the Fe film below $10 \mathrm{ML}$, both of which 
suggest that the enhancement of the orbital moment is not caused by the reduced symmetry of the interfaces. Since that film was grown at room temperature, unlike the present sample, the orbital moment enhancement may have been induced by randomly outdiffused elements from the substrate, as indicated by Giovanelli et al. ${ }^{29}$ and thus did not necessarily support PMA. Besides, their Fe film is capped by Co layer whose effects on the magnetism, especially the anisotropy of the Fe film, is not yet clear.

The other notable feature of the sample grown at low temperature is the unprecedented low ferromagnetic onset thickness of $\sim 2.1$ ML. The lowest ferromagnetic onset thickness previously reported ${ }^{12}$ was $\sim 2.75 \mathrm{ML}$ with $T_{C}$ $\sim 100 \mathrm{~K}$. Furthermore, they predicted that $T_{C}$ would reach $0 \mathrm{~K}$ at $2.5 \mathrm{ML}$ by extrapolating their results on the thickness dependence of $T_{C}$. The early ferromagnetic onset seems to have been caused by the low-temperature growth in the following way. The suppression of the outdiffusion would result in the virtual absence of $\mathrm{Fe}$ compounds that are mostly nonmagnetic or have much-reduced magnetic moments. Hence, extended ferromagnetic $\mathrm{Fe}$ domains can form without the inclusion of $\mathrm{Fe}$ compounds that disturb the long-range ferromagnetic order and delay the ferromagnetic onset.

\section{SUMMARY AND CONCLUSION}

Both XMCD and MOKE studies have revealed the magnetism of virtually pristine $\mathrm{Fe}$ films grown on $\mathrm{GaAs}(100)$ at $\sim 80 \mathrm{~K}$. We found that the 2.1-ML-thick Fe film clearly shows PMA, which is observed over a very limited thickness range of around 2.1 ML. The SRT from PMA to the in-plane magnetic anisotropy occurs at $\sim 2.8 \mathrm{ML}$.

The observed magnetic features are mainly caused by the suppression of both Ga and As migration from the substrate, especially the latter. This preserves surface anisotropy and allows the observation of perpendicular ferromagnetic anisotropy. The observed PMA is associated with the enhanced orbital moment by $\sim 50 \%$, which is about as much as is usually observed for magnetic films at their ultrathin limit, but is in disagreement with some previous reports. ${ }^{11}$ In conclusion, the pristine Fe film on GaAs(100) seems to be simply an Fe film with no anomalous properties. It should be interesting to examine whether the kinetic stabilization and observation of PMA of Fe film is feasible on GaAs surfaces other than Ga-terminated (100), the present one.

\section{ACKNOWLEDGMENTS}

This study was supported by KOSEF through CSCMR (S.-J.O. and J.-S.K.) and The work at POSTECH (J.-S.K. and J.-H.P) is supported by KRF Grant No. KRF-2006-312C00523 and POSTECH research fund. *jskim@sookmyung.ac.kr

${ }^{1}$ J. R. Waldrop and R. W. Grant, Appl. Phys. Lett. 34, 630 (1979).

${ }^{2}$ H. J. Zhu, M. Ramsteiner, H. Kostial, M. Wassermeier, H.-P. Schönherr, and K. H. Ploog, Phys. Rev. Lett. 87, 016601 (2001).

${ }^{3}$ S. A. Chambers, F. Xu, H. W. Chen, I. M. Vitomirov, S. B. Anderson, and J. H. Weaver, Phys. Rev. B 34, 6605 (1986).

${ }^{4}$ E. Kneedler, P. M. Thibado, B. T. Jonker, B. R. Bennett, B. V. Shanabrook, R. J. Wagner, and L. J. Whitman, J. Vac. Sci. Technol. B 14, 3193 (1996).

${ }^{5}$ S. C. Erwin, S.-H. Lee, and M. Scheffler, Phys. Rev. B 65, 205422 (2002).

${ }^{6}$ S. Mirbt, S. Sanyal, C. Isheden, and B. Johansson, Phys. Rev. B 67, 155421 (2003).

${ }^{7}$ A. Filipe, A. Schuhl, and P. Galtier, Appl. Phys. Lett. 70, 129 (1997)

${ }^{8}$ E. M. Kneedler and T. Jonker, J. Appl. Phys. 81, 4463 (1997).

${ }^{9}$ M. Przybylski, S. Chakraborty, and J. Kirschner, J. Magn. Magn. Mater. 234, 505 (2001)

${ }^{10}$ Y. B. Xu, E. T. M. Kernohan, D. J. Freeland, A. Ercole, M. Tselepi, and J. A. C. Bland, Phys. Rev. B 58, 890 (1998).

${ }^{11}$ J. S. Claydon, Y. B. Xu, M. Tselepi, J. A. C. Bland, and G. van der Laan, Phys. Rev. Lett. 93, 037206 (2004).

${ }^{12}$ F. Bensch, R. Moosbühler, and G. Bayreuther, J. Appl. Phys. 91, 8754 (2002).

${ }^{13}$ O. Thomas, Q. Shen, P. Schieffer, N. Tournerie, and B. Lepine, Phys. Rev. Lett. 90, 017205 (2003).

${ }^{14}$ G. Wastlbauer and J. A. C. Bland, Adv. Phys. 54, 137 (2005).

${ }^{15}$ G. W. Anderson, M. C. Hanf, and P. R. Norton, Phys. Rev. Lett. 74, 2764 (1995).

${ }^{16}$ B. D. Schultz, H. H. Farrell, M. M. R. Evans, K. Lüdge, and C. J.
Palmstrøm, J. Vac. Sci. Technol. B 20, 1600 (2002).

${ }^{17}$ Y. Chye, V. Huard, M. E. White, and P. M. Petroff, Appl. Phys. Lett. 80, 449 (2002).

${ }^{18}$ M. Marangolo, F. Gustavsson, M. Eddrief, Ph. Sainctavit, V. H. Etgens, V. Cros, F. Petroff, J. M. George, P. Bencok, and N. B. Brookes, Phys. Rev. Lett. 88, 217202 (2002).

${ }^{19}$ F. Zavaliche, W. Wulfhekel, and J. Kirschner, Phys. Rev. B 65 , 245317 (2002).

${ }^{20}$ J.-M. Lee, S.-J. Oh, K. J. Kim, S.-U. Yang, J.-H. Kim, and J.-S. Kim, Phys. Rev. B 75, 125421 (2007).

${ }^{21}$ T. C. Kim, J.-M. Lee, Y. S. Kim, D. Y. Noh, S.-J. Oh, and J.-S. Kim, arXiv:cond-mat/0603123 (unpublished).

${ }^{22}$ E. M. Kneedler, B. T. Jonker, P. M. Thibado, R. J. Wagner, B. V. Shanabrook, and L. J. Whitman, Phys. Rev. B 56, 8163 (1997).

${ }^{23}$ B. T. Thole, P. Carra, F. Sette, and G. van der Laan, Phys. Rev. Lett. 68, 1943 (1992).

${ }^{24}$ P. Carra, B. T. Thole, M. Altarelli, and X. Wang, Phys. Rev. Lett. 70, 694 (1993).

${ }^{25}$ C. T. Chen, Y. U. Idzerda, H.-J. Lin, N. V. Smith, G. Meigs, E. Chaban, G. H. Ho, E. Pellegrin, and F. Sette, Phys. Rev. Lett. 75, 152 (1995).

${ }^{26}$ M. Kosuth, V. Popescu, H. Ebert, and G. Bayreuther, Europhys. Lett. 72, 816 (2005).

${ }^{27}$ M. Marangolo, F. Gustavsson, G. M. Guichar, M. Eddrief, J. Varalda, V. H. Etgens, M. Rivoire, F. Gendron, H. Magnan, D. H. Mosca, and J.-M. George, Phys. Rev. B 70, 134404 (2004).

${ }^{28}$ J. S. Claydon, Y. B. Xu, M. Tselepi, J. A. C. Bland, and G. van der Laan, J. Appl. Phys. 95, 6543 (2004).

${ }^{29}$ L. Giovanelli, G. Panaccione, G. Rossi, M. Fabrizioli, C.-S. Tian, P. L. Gastelois, J. Fujii, and C. H. Back, Phys. Rev. B 72, 045221 (2005) 